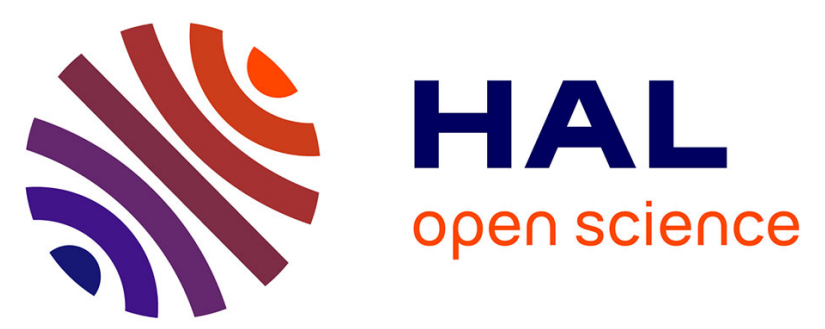

\title{
DIFFUSION ACOUSTIQUE PAR UN TUBE EN INCIDENCE OBLIQUE. RAYONNEMENT EN BOUT DE TUBE
}

\author{
F. Leon, G. Maze
}

\section{> To cite this version:}

F. Leon, G. Maze. DIFFUSION ACOUSTIQUE PAR UN TUBE EN INCIDENCE OBLIQUE. RAYONNEMENT EN BOUT DE TUBE. Journal de Physique IV Proceedings, 1992, 02 (C1), pp.C1-709C1-712. 10.1051/jp4:19921153 . jpa-00251112

HAL Id: jpa-00251112

https://hal.science/jpa-00251112

Submitted on 1 Jan 1992

HAL is a multi-disciplinary open access archive for the deposit and dissemination of scientific research documents, whether they are published or not. The documents may come from teaching and research institutions in France or abroad, or from public or private research centers.
L'archive ouverte pluridisciplinaire HAL, est destinée au dépôt et à la diffusion de documents scientifiques de niveau recherche, publiés ou non, émanant des établissements d'enseignement et de recherche français ou étrangers, des laboratoires publics ou privés. 


\title{
DIFFUSION ACOUSTIQUE PAR UN TUBE EN INCIDENCE OBLIQUE. RAYONNEMENT EN BOUT DE TUBE
}

\author{
F. LEON et G. MAZE \\ Université du Havre, L.A.U.E. - U.R.A. C.N.R.S. 1373, Place Robert Schuman, F-76610 Le Havre, \\ France
}

\begin{abstract}
The waves generated on a determined area of a cylindrical shell and with an angle of incidence $\alpha$ are propagated parallel with the axis of this cylinder. Then, the radiation. in the far field, can be observed in the end of this shell with a quasi-harmonic method. The axial displacement is analysed and the use of the Rayleigh integral allows us to determine the radiated pressure. The results of the theoretical calculation are in agreement with the results of experiments.
\end{abstract}

les études expérimentales et théoriques de la diffusion acoustique par des tubes, remplis d'air et immergés dans l'eau, mettent en évidence la propagation de deux types d'ondes : les ondes de galerie à écho (ondes circonférentielles) et les ondes guidées. La diffusion acoustique par des coques cylindriques (de longueur supposée infinie) a été examinée en faisant varier la direction d'insonation par rapport à la normale à l'axe de la cible. L'étude particulière d'une coque d'aluminium, dont le rapport entre le rayon interne (b) et le rayon externe (a) est égale à 0,84 , montre le caractère propagatif des ondes observées aux fréquences de résonance à l'aide d'un montage bistatique. A ces fréquences, les ondes générées sur une zone déterminée de la cible et sous un angle d'incidence $\alpha$ se propagent parallèlement à l'axe du cylindre et réémettent leur énergie, au cours de leur propagation, dans une direction d'angle $\alpha$ avec la normale au point de réemission. Dans le cas de cette cible, un tel montage (Fig.1 : position (1)) permet de relever séparément, pour un angle $\alpha$, le spectre de résonances de l'onde de galerie à écho $(\mathrm{l}=2)$ et celui de l'onde guidée $(\mathrm{p}=1)$ grâce à leurs vitesses de propagation différentes.

L'insonation de cette cible sur une zone déterminée provoque donc, aux fréquences de résonance. la propagation d'ondes le long du cylindre. Il est alors possible d'observer en champ lointain le rayonnement en bout du tube (section circulaire) à l'aide d'une méthode bistatique quasi-harmonique (Fig.1 : position (2)). Cette cible est supposée de longueur semi-infinie afin qu'il ne puisse pas se produire un phénomène d'ondes stationnaires (cas de tubes de longueur limitée).

ETUDE THEORIQUE.

Cette étude est fondée sur la détermination d'un modèle simple de la pression rayonnée à l'extrémité d'un tube. Pour cela, on considère que la surface de cette extrémité est supposée animée d'un mouvement caractérisé par son déplacement normal ponctuel $\mathrm{U}_{\mathrm{Z}}(\mathrm{M}, \mathrm{t})$ (point $\mathrm{M}$ appartenant à la section $\mathrm{S}$ du tube). L'axe de la cible cylindrique est l'axe $\mathrm{z}$ du système de coordonnées cylindriques. 


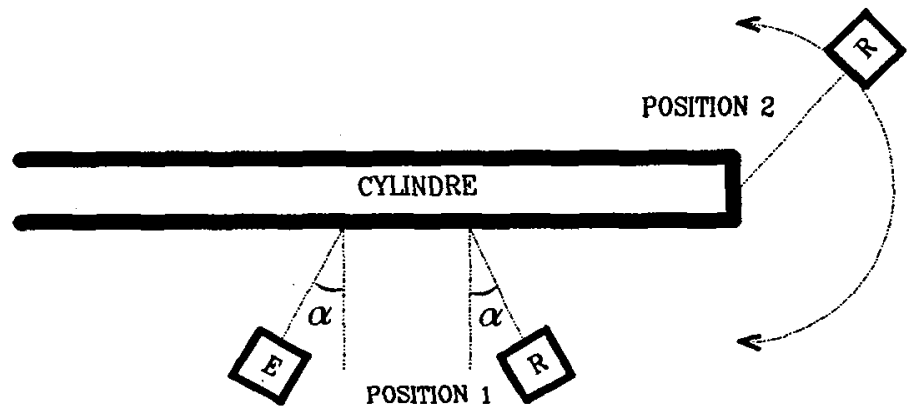

Fig.1 : Positions du transducteur-recepteur selon l'expérience réalisée.

En utilisant les informations présentées dans [1], il est alors possible d'écrire, lors d'une excitation forcée, l'élongation axiale dans ce tube immergé dans l'eau et contenant de l'air sous la forme:

$U_{z}=\frac{\partial \phi}{\partial z}+\frac{A_{\theta}}{r}+\frac{\partial A_{\theta}}{\partial r}-\frac{1}{r} \frac{\partial A_{r}}{\partial \theta}=\sum_{n=0}^{\infty} U_{z n}$

$\phi \quad$ étant le potentiel scalaire représentant les ondes longitudinales et $\quad \overrightarrow{\mathbf{A}}\left(\mathbf{A}_{r}, \mathbf{A}_{\theta}, \mathbf{A}_{r}\right)$ étant un potentiel vecteur représentant les ondes transversales.

A partir de cette formule, il est possible de calculer et de représenter le module du déplacement $\mathrm{U}_{\mathrm{Z}}$ sur une section du tube située dans la zone d'excitation forcée. Cette représentation (symétrie des déplacements par rapport à l'axe ox) se fait sous forme de courbes de niveau (Fig.2a). Afin de faciliter le traitement graphique des données, la valeur maximale du module de déplacement a été égalisée à 1. Ces différentes courbes de niveau caractérisant le module de déplacement sont alors tracées en prenant un intervalle de calcul de 0,1. L'observation de l'exemple cité (Fig.2a) a montré une disposition particulière des courbes de niveau. Le tracé du déplacement $\mid U_{\mathrm{z}} \mathrm{l}$ à mi-épaisseur (Fig.2b) donne un nombre de maximums égale au mode de la résonance sur une demi-section de la cible.

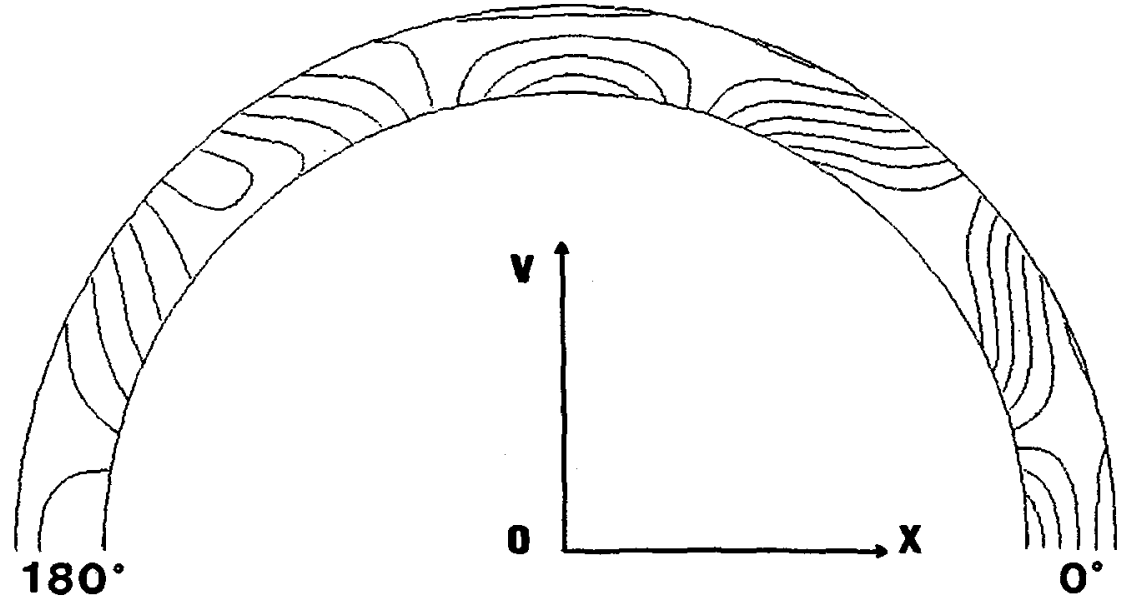

Fig.2a : Représentation du module de déplacement $\mid U_{Z} l$ sous forme de courbes de niveau $\alpha=10^{\circ}$. Onde de type guidée $(p=1)$. Fréquence $=397 \mathrm{kHz}$, Mode $=2$ 


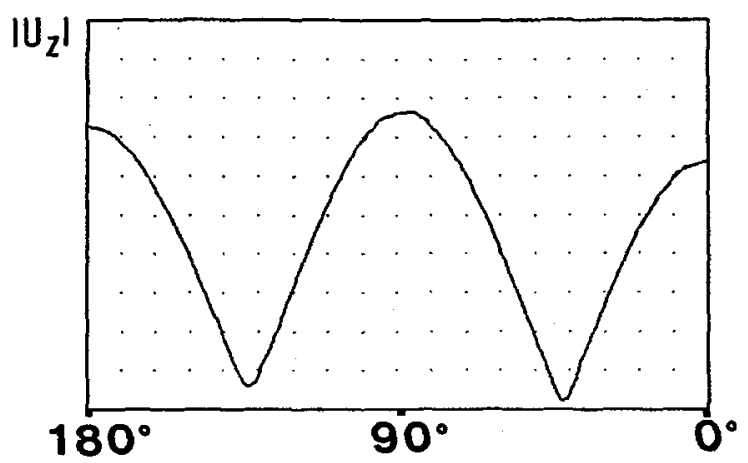

Fig.2b : Module du déplacement $\left|U_{\mathrm{z}}\right|$ à mi-épaisseur du tube.

Le tube étant insoné sur une zone fixée, les ondes générées aux fréquences de résonance se propagent parallèlement à l'axe du cylindre. A ces fréquences, il faut alors déterminer un modèle simple de la pression rayonnée en champ lointain à l'extrémité de celte cible (Fig.1 : position (2)). Dans ce cas, seul la contribution du déplacement $U_{z n}$ d'indice $n$ (correspondant au mode de résonance) est prise en compte. La pression rayonnée $P_{r}$ est définie en utilisant une expression intégrale. Pour cela, on considère le cas du rayonnement dans le demi-espace extérieur infini (milieu fluide) où l'on vérifie la condition de Sommerfeld à l'infini. On suppose alors que la section circulaire (S) à l'extrémité du tube vibre et rayonne en fonction de l'élongation axiale $U_{\mathrm{zn}}(\mathrm{M}, \mathrm{t})$. La pression rayonnée $P_{r}$ (point d'observation en 0 ) est alors exprimée par l'intégrale de Rayleigh [2-3] d'où on en déduit la relation suivante :

$$
P_{r}(0)=-\frac{1}{2 \pi} \int_{0}^{2 \pi} \int_{b}^{a} \rho \omega^{2} U_{z n}(M) G(0, M) d S
$$

dans laquelle $\rho$ est la masse volumique de l'eau, $\omega$ est la fréquence angulaire et $G(0, M)$ est la fonction de Green. Les figures 3a et 4a montrent deux résultats obtenus à l'aide de cette intégrale.

RESULTATS EXPERIMENTAUX.

Dans cet article, l'expérimentation a été réalisée à l'aide d'un tube d'aluminium (diamètre : $6 \mathrm{~mm}$. longueur : $1 \mathrm{~m}$ ) rempli d'air dont les extrémités étaient obturées par un bouchon de mastic peu épais. Les deux transducteurs et la cible étant placés dans un plan horizontal, le transducteurrécepteur effectue une rotation de $180^{\circ}$ à l'extrémité de la cible (Fig.1 : position (2)) afin de relever la pression rayonnée. Le tube est insoné par des trains de sinusoïdes dont la durée est égale à $300 \mu \mathrm{s}$ afin d'établir un état stationnaire sur la circonférence et propagatif parallèlement à l'axe du cylindre. Au préalable. la fréquence de résonance expérimentale est fixée avec précision à l'aide du montage de la figure 1 (position (1)).

Dans le cas des deux exemples théoriques précédemment cités, l'expérimentation donne pour résultats les diagrammes angulaires des figures $3 \mathrm{~b}$ et $4 \mathrm{~b}$. La comparaison de ces figures montre un bon accord entre les résultats théoriques et expérimentaux si on ne tient pas compte des lobes latéraux ( $t$ ) obtenus expérimentalement. On constate que, pour un angle d'incidence $\alpha$ fixé. la position angulaire de ces lobes latéraux varie peu en fonction du mode. Par contre, pour un mode $n$ fixé, la position angulaire de ces lobes varie en fonction de l'angle d'incidence. Ces lobes semblent être dus à un effet de bord produit, lors d'une résonance, par l'élongation radiale, cette dernière n'étant pas prise en compte dans le calcul théorique. 


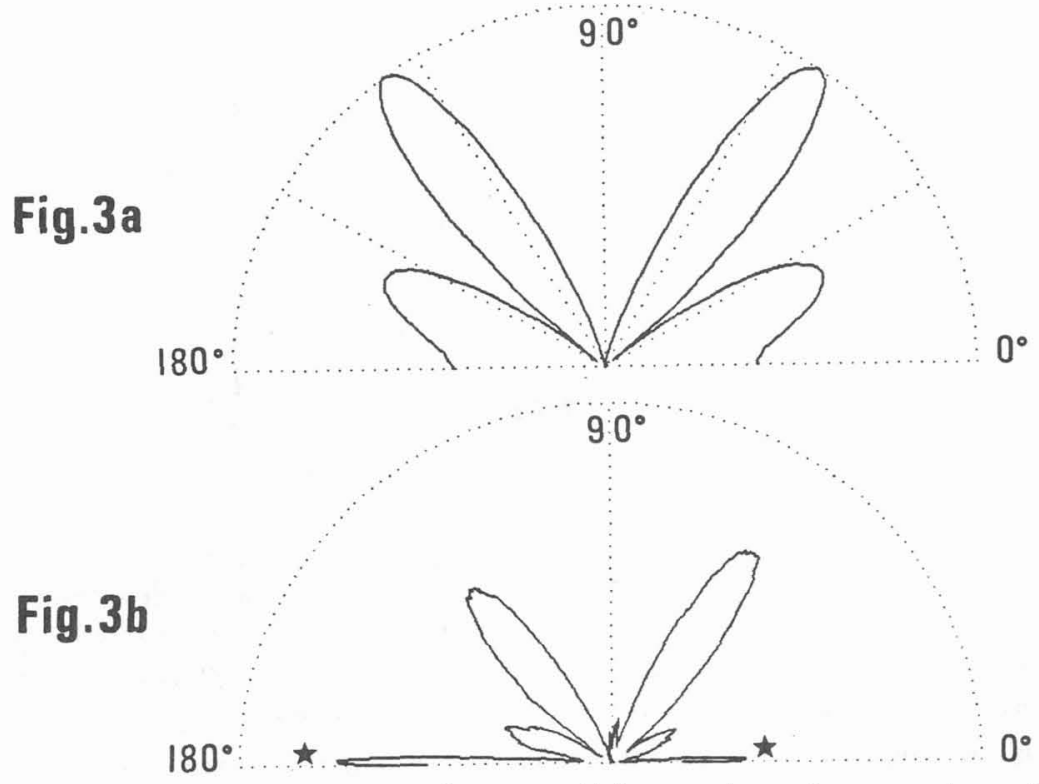

Fig.3a : Pression rayonnée théorique : $\alpha=2 ;, F=1059 \mathrm{kHz}$, onde guidée $: p=1$, mode $N=6$

Fig.3b : Pression rayonnée expérimentale : $\alpha=2 \cdot F=1067 \mathrm{kHz}$, onde guidée : $p=1$, mode $N=6$

$9: 0^{\circ}$

Fig.4a

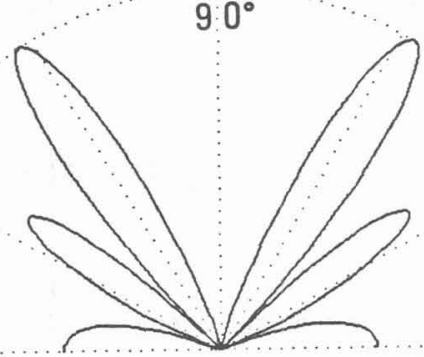

$0^{\circ}$

$90^{\circ}$

Fig.4b

$180^{\circ}$

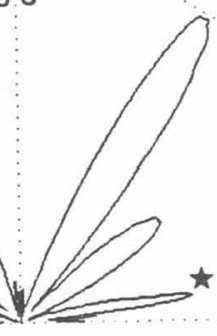

$0^{\circ}$

Fig.4a : Pression rayonnée théorique : $\alpha=10, \mathrm{~F}=1330 \mathrm{kHz}$, onde guidée $: \mathrm{p}=1$, mode $\mathrm{N}=7$

Fig.4b : Pression rayonnée expérimentale : $\alpha=10 \cdot \mathrm{F}=1349 \mathrm{kHz}$, onde guidée $: \mathrm{p}=1$, mode $\mathrm{N}=7$

Ce travail est soutenu par la DRET (contrat $N \cdot 90 / 182$ ).

[1] Fernand LEON, Florence LECROQ, Dominique DECULTOT and Gérard MAZE, Scattering of an obliquely incident acoustic wave by an infinite hollow cylindrical shell. J.Acoust.Soc.Amer., article accepté.

[2] H.A. SCHENCK, Improved integral formulation for acoustic radiation problems, J.A.S.A. 44 (1), 1967.

[3] P.M. MORSE, K.U. INGARD, Theoretical Acoustics, McGraw-Hill Inc., 1968. 